\title{
Learn to Move: Activity Specific Motion Models for Tracking by Detection ${ }^{\star}$
}

\author{
Thomas Mauthner, Peter M. Roth, and Horst Bischof \\ Inst. f. Computer Graphics and Vision, \\ Graz University of Technology, Austria
}

\begin{abstract}
In this paper, we focus on human activity detection, which solves detection, tracking, and recognition jointly. Existing approaches typically use off-the-shelf approaches for detection and tracking, ignoring naturally given prior knowledge. Hence, in this work we present a novel strategy for learning activity specific motion models by feature-totemporal-displacement relationships. We propose a method based on an augmented version of canonical correlation analysis (AuCCA) for linking high-dimensional features to activity-specific spatial displacements over time. We compare this continuous and discriminative approach to other well established methods in the field of activity recognition and detection. In particular, we first improve activity detections by incorporating temporal forward and backward mappings for regularization of detections. Second, we extend a particle filter framework by using activity-specific motion proposals, allowing for drastically reducing the search space. To show these improvements, we run detailed evaluations on several benchmark data sets, clearly showing the advantages of our activity-specific motion models.
\end{abstract}

\section{Introduction}

In the recent past, human action recognition has been of growing interest in Computer Vision, where typical applications include visual surveillance, human computer interaction, and monitoring systems for elderly people. Thus, a variety of approaches have been proposed introducing new features, representations, or classification methods. Since actions can be described as chronological sequences special attention has been paid on how to incorporate temporal information into the feature description. For the basic problem of activity classification per video, global representations are a common choice [12. Different spatio-temporal interest points (STIP) and descriptors were evaluated in [1]; the relationship between spatial and temporal STIPs was additionally explored in [2]. This applies for early datasets with homogeneous backgrounds and single moving objects like [3] as well as for recently collected datasets showing a larger variety of the activities, e.g, 4.

\footnotetext{
* The work was supported by the Austrian Research Promotion Agency (FFG) project SHARE in the IV2Splus program and the Austrian Science Fund (FWF) under the project MASA (P22299).
}

A. Fusiello et al. (Eds.): ECCV 2012 Ws/Demos, Part III, LNCS 7585, pp. 183-192, 2012.

(C) Springer-Verlag Berlin Heidelberg 2012 
The problem gets even harder if additionally to classification also detection is considered. This is of relevance if, e.g., several simultaneous activities, background motion, or high inner class variability of backgrounds have to be handled. Previous methods either assumed given tracks from, e.g., background subtraction or general tracking approaches [56] and analyzed activities per track or just per frame 78 . For linking the latter one in an online manner, particle filter is a favored choice [7,5. Recently, an offline linking method via graph-based representation has been proposed by [8]. But in general, all these approaches do not take into account the prior knowledge about the correlation of motion and specific activities.

Hence, we propose to learn activity specific motion models in a combined recognition and temporal voting scheme. In particular, we incorporate a temporal displacement voting, which is learned via an augmented canonical correlation analysis (AuCCA), into a regularization for activity detections and a particle filter framework. We compare the thus obtained results to state-of-the-art methods for combined activity classification and voting, namely k-means hierarchies and Hough forest. The main contributions of this paper are:

- an efficient classification and voting framework via AuCCA

- the correction of activity detections via consistency checks

- a particle filter tracker with activity specific motion proposals.

The reminder of the paper is organized as follows. In Section 2 , we first define the problem of learning combined classification and temporal displacement voting. We propose our AuCCA approach and show how the idea of temporal displacement voting can be incorporated into other standard learning frameworks. Next, Section 3 depicts the detection and tracking framework and shows how to incorporate the learned temporal motion models. Experimental results are shown in Section 4, where competitive results for activity classification are presented. Moreover, we demonstrate the advantages of learned motion models for unsupervised detection correction and evaluate tracking vs. random-walk and optical flow based models. Finally, Section 5 summarizes the proposed approach and gives an outlook on how AuCCA can be incorporated into other concepts for temporal activity detection linking.

\section{Learning of Specific Motion Models}

First of all, we describe the general idea of learning motion models via temporal displacements. To allow for combined activity detection/classification we describe an action by a d-dimensional feature vector $x_{i} \in \mathbb{R}^{d}$ in a temporal context. Thus, in addition to the corresponding class labels $y_{i} \in\{1,2, \ldots, c\}$ also the temporal offsets for the activity center within in $t+\tau$ frames are given: $\mathbf{d}_{i}{ }^{\tau} \in \mathbb{R}^{2}$. Thus, during training we have a prior knowledge on the object's movement within $\tau$ frames.

In the following, we determine three approaches that can be adopted for that purpose: (a) hierarchical k-means clustering, (b) Random Forest (RF), and (c) 
Canonical Correlation Analysis (CCA). K-means is an unsupervised generative method not exploiting class labels during training; however, class labels and displacement values can be stored according to the obtained clusters. In contrast, Random Forest (RF) use a discriminative splitting function and can also be extended to train according to displacement values 9. Canonical Correlation Analysis (CCA) additionally allows for maximizing the correlation between input feature vectors, class labels, and displacement vectors. We start the discussion with the proposed AuCCA exploiting the correlation between the feature vectors and the displacement vectors. Then, for comparison, we extend k-means and RF such that they can also learn the displacement values.

\subsection{Augmented Canonical Correlation Analysis (AuCCA)}

In general, the goal of Canonical Correlation Analysis (CCA) is to find pairs of directions that maximize the correlation between two random variables [10]. Formally, given two mean normalized random variables $\mathbf{x}$ and $\mathbf{d}, \mathrm{CCA}$ is defined as the problem of finding a set of two basis vectors $\mathbf{w}_{x}$ and $\mathbf{w}_{d}$ such that the correlation between the projections $x^{\prime}=\mathbf{W}_{x}^{\top} \mathbf{x}$ and $d^{\prime}=\mathbf{W}_{d}^{\top} \mathbf{d}$ is maximized. These are obtained by maximizing the correlation coefficient

$$
\rho=\frac{\mathbf{w}_{x}^{\top} \mathbf{C}_{x d} \mathbf{w}_{d}}{\mathbf{w}_{x}^{\top} \mathbf{C}_{x x} \mathbf{w}_{x} \mathbf{w}_{d}^{\top} \mathbf{C}_{d d} \mathbf{w}_{d}},
$$

where $\mathbf{C}_{x x}$ and $\mathbf{C}_{d d}$ are the within-class covariance matrices and $\mathbf{C}_{x d}$ is the between-class covariance matrix. The projections onto $\mathbf{w}_{x}$ and $\mathbf{w}_{d}$, i.e., $x^{\prime}$ and $d^{\prime}$, are called the canonical factors. In our case, we build on an efficient and numerically stable formulation building on an SVD decomposition [11.

Assuming that all input features in $\mathbf{X} \in \mathbb{R}^{d \times n}$ and the corresponding displacement values in $\mathbf{D} \in \mathbb{R}^{2 \times n}$ are given, we can project the training data onto their canonical correlation coefficients: $\mathbf{X}^{\prime}=\mathbf{W}_{x}^{\top} \mathbf{X}$, with $\mathbf{X}^{\prime} \in \mathbb{R}^{2 \times n}$. We estimate a linear mapping $\mathbf{F}$ from the canonical correlation coefficients to the corresponding displacement values $\mathbf{D}$ using the least square solution of $\mathbf{F}=\mathbf{D X}^{\prime \top}$. This yields a very efficient representation as only $\mathbf{W}_{x} \in \mathbb{R}^{2 \times d}$ and $\mathbf{F} \in \mathbb{R}^{2 \times 2}$ have to be stored for later evaluations. During testing we estimate the displacements for a given sample vector $\hat{\mathbf{x}}$ by projection onto the canonical space by $\hat{\mathbf{x}}^{\prime}=\mathbf{W}_{x} \hat{x}$ and the mapping $\hat{\mathbf{d}}=\mathbf{F} \hat{\mathbf{x}}^{\prime}$.

The dimensionality of the canonical correlation coefficients is limited by the smaller dimensionality of the correlated training sets $\mathbf{X}$ and $\mathbf{D}$. Thus, too much discriminative information could get lost due to reduced dimensionality. To overcome this problem, Kernel-CCA can be used, which, however, would be computationally much more expensive. Instead, we exploit the additional information given by a multi-class problem and augment the displacement values in each $\mathbf{d}_{i}$ with a binary label vector $\mathbf{y}_{i}$ with $\mathbf{y}_{i}(j)=1$ for the correct class and 0 otherwise.

The incorporated class information strengthens the correlation between points and the higher dimensional correlation space allows for better representation of the mapping from feature to offset space. Moreover, we get a classifier for 
free. Fig. 1 depicts the distribution of temporal offsets over all activities in the UCF-Sports dataset. The coefficients of standard CCA show directly the distribution in $d=2$ dimensional space before multiplying with $\mathbf{F}$. The $d=(c+2)$ dimensional coefficients of AuCCA have been visualized using a metric multidimensional down-scaling to a dimensionality of 2 . Due to the high dimensionality of the coefficient space, the spatial offsets cannot directly be seen, but the class specific grouping of AuCCA is demonstrated. Illustrative examples for displacement vectors are shown in Fig. 2.
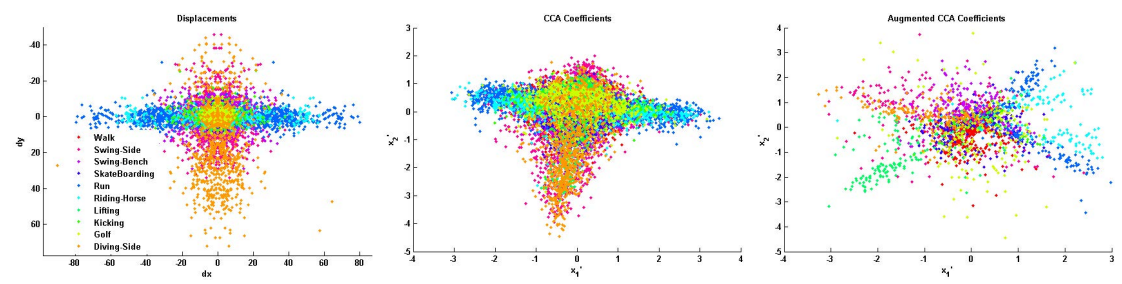

Fig. 1. Distribution of temporal offsets: left: displacement values for UCF sports dataset, color-coded for individual actions; middle: standard 2-dimensional CCA coefficients; right: coefficients of AuCCA, nicely showing the separated classes

\subsection{Hierarchical K-Means Clustering}

The key idea of hierarchical k-means clustering, also known as Vocabulary Tree, is to recursively split the training data via $\mathrm{k}$-means clustering. This process is repeated recursively until no samples are left in a branch of the hierarchy or the maximum depth $L$ is reached. For each of the thus obtained cluster centers $\varphi$ (i.e., leaf nodes) we can then estimate a class probability distribution $p(c \mid \varphi)$. To learn action specific motion models, we additionally store temporal displacement vectors of each training sample in each leaf node $\varphi$.

During evaluation, a test sample is traversed down the tree, using depth-firstsearch, until it reaches a leaf node. Hence, for each sample we get the class probabilities and the temporal displacement values stored in the leaf node. For simplicity we average the displacement values and report the mean $d_{x}, d_{y}$ values. An important factor for good generalization and robustness is the use of $\mathrm{k}$-means ensembles, i.e., we split the training set randomly into $T$ subsets, and train $T$ individual k-means hierarchies. Similar to the idea of Random Forests [129], this avoids overfitting on the training data and shows better generalization capabilities. During evaluation, a test sample is traversing down all hierarchies and the results of all reached leaves are averaged.

\subsection{Random Forests}

Hierarchical k-means clustering can be adopted for the intended task by additionally storing the displacement vectors in the leaf nodes. However, the discriminative information given by the class labels is ignored. To also exploit this 
information Random Forests can be used. In general, a forest consists of an ensemble of $T$ binary decision trees, which are constructed recursively starting from the root node. For each node, binary tests are computed to split the data $\mathbf{X}$ into subsets going to the left or the right branch [13.

Two prominent ways to build a Random Forest are to maximize the information gain [12139] or in addition to minimize the displacement uncertainty (as proposed for the Hough Forest 9]). Maximizing the information gain is equivalent to minimizing the class-label uncertainty. In the second case, samples with similar displacements are grouped together. The decision whether the class-label or displacement uncertainty should be minimized is selected randomly during training. For evaluation, a test sample is traversing down each tree and the results of all reached leaves are averaged. Thus, in contrast to the previous mentioned k-means we have a supervised and discriminative discretization of our feature space.

\section{Framework (Building Action Tracks)}

This section delineates how the proposed activity-specific motion models learned with AuCCA can be exploited for the task of specific detection and tracking.

\subsection{Detection Correction with Forward-Backward Consistency Regularization}

The concept of forward-backward mapping is known from key-point tracking or optical flow estimation. Estimating the forward displacement of a small and local image patch $t \rightarrow t+1$ should deliver the same result as tracking backward $t+1 \rightarrow t$. Section 2 formalized how temporal voting information can be learned for complete object patches, where we are not limited to $t+1$ displacements but can train an arbitrary mapping backward for $\tau$ and $-\tau$ frames. Now, we exploit this knowledge to filter out false-positive detections by applying AuCCA displacement mappings for positive detections at time $t$. We estimate a forward mapping of $\tau$ frames, run the detector at the thus proposed positions at $t+\tau$, and map positive detections backward with AuCCA learned for $-\tau$ displacements.

To show the robustness and the generalization capabilities, results from tracking on Weizmann robust data are illustrated in Fig. 2. Green and red points visualize positive detections for time $t$ and $t+\tau$, respectively, and colored lines the temporal votings in $\tau=5$ frames. We can see an accurate voting to the object's center in $+\tau$ frames and how false positive detections are marginalized out by missing backward mappings. We derive a conservative learning framework from this observation for unsupervised mining of new positive and negative samples. We are regularizing over the spatial and temporal neighborhood of positive detections. Detections with no forward-backward consistent detection in their local neighborhood are defined as new negative samples. Detections with a majority of consistent neighbors are defined as new positives while others are seen as neutral and are not used for updating the detector (see Fig. 3). 

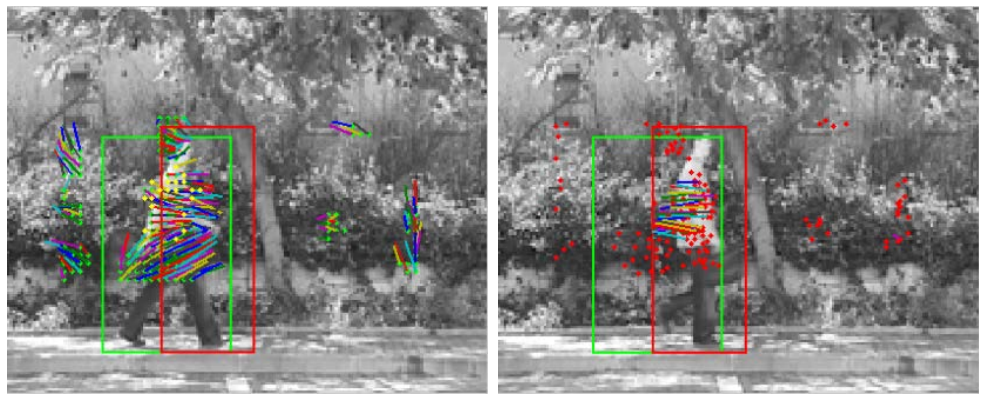

Fig. 2. Positive detections in frame $t$ with AuCCA forward displacement vectors for $t+\tau$ (left). Detector evaluation of proposed positions at $t+\tau$ and backward displacements for positive detections with consistent backward mapping (right). Green and red bounding boxes show the ground-truth for time $t$ and $t+\tau$, respectively.

\subsection{Review of Particle Filter}

Particle filtering for tracking [14] provides a probabilistic framework, which maintains multiple hypotheses of the current object state and has proven to yield robust tracking results. The probability distribution of the hidden target state $\mathbf{s}_{t}$ of the tracked object at time step $t$ is estimated using a set of $N_{P}$ weighted particles $S_{t}=\left\{s_{t}^{i}, w_{t}^{i}\right\}$ with $i=1 \ldots N_{P}$ at time step $t$ and the associated measurements $z_{t}^{i}$. Each particle $x_{t}^{i}$ simulates the real hidden state of the object, using the dynamic model $p\left(s_{t}^{i} \mid s_{t-1}^{i}\right)$ and the observation likelihood $p\left(z_{t}^{i} \mid s_{t}^{i}\right)$. The object state is approximated by a weighted average over this finite set of particles. To avoid a degeneration of the particle set, a re-sampling of the particles is necessary according to their particle weights $w_{t}^{i} \approx p\left(z_{t}^{i} \mid s_{t}^{i}\right)$. For more details we would refer to [14.

\subsection{Integrating Intelligent Motion Models}

The most important parameters are the number of particles $N_{P}$ and the choice of the dynamic model $p\left(s_{t}^{i} \mid s_{t-1}^{i}\right)$. The better the motion model the less particles are needed and a small re-sampling rate would indicate a good fit. In addition, the efficiency is increased as the runtime is linear according to the number of particles. A classical choice for the dynamic model would be to use random walks (e.g., used for activity tracking in [5]), where the motion is modeled by Gaussian noise: $s_{t-1}^{i}+\mathcal{N}\left(0, \sigma_{t}\right)$. Obviously this is very inefficient as no information about the current object motion is incorporated, but a general choice if nothing about the object's behavior is known. A slightly advanced version is to use a constant velocity model, where in addition to Gaussian noise the velocity $(u, v)$ at time $t-1$ is added to move particles to $s_{t}[7]$.

None of the previous mentioned approaches takes into account that during tracking of activities prior knowledge about activity specific motion is given. In addition, particle filtering is perfectly suited for incorporating an individual 
motion model per $s_{t-1}^{i}$. Given the learning frameworks presented in Section 2 we can directly integrate their temporal displacement proposals into the motion model. For each particle state $s_{t-1}^{i}$, describing a potential position, we can extract a feature vector $\mathbf{x}_{t-1}^{i}$ and evaluate it using AuCCA, which yields a displacement suggestions $\hat{\mathbf{d}}_{i}$. If required, also the class labels $\hat{\mathbf{y}}_{i}$ can be estimated. The transition to $s_{t}^{i}$ is modeled by $\hat{\mathbf{d}}_{i}+\mathcal{N}\left(0, \sigma_{d}\right)$ (in our case we have $\sigma_{d}<<\sigma_{t}$ ), which is mainly needed for diffusing particle positions after resampling. A corresponding evaluation and a comparison to optical flow and random-walk based motion models is given in the experimental section.

\section{Experiments}

We selected two commonly known datasets for evaluation of our framework, i.e., Weizmann 1 data set [3] and UCF-Sports [4]. Both datasets allow for object centered recognition and contain activities with different or alternating motion directions. Please note that we our primary goal was not breaking recognition scores. Especially for the UCF-Sports data set global representations on video level show superior results [1]. But this is somehow misleading as the activity defining the label of a video may only occupy a fraction of the video volume. Therefore, global representations are often influenced more by the global motion and a small variability of the background than by the activity itself. Hence, such representations often "solve the dataset" but do not solve the task. We are interested in exploiting the object's motion related to its current activity and in generating detection tracklets throughout videos. We compensate for global camera motion to estimate the real objects motion for training and testing. Otherwise the object centered camera motion gives a too strong prior to evaluate for displacement estimation. Note that for tracking our proposed motion estimation could be combined with global motion in the same way as shown in [7].

\subsection{Evaluation of Complexity}

We start with a general evaluation of the compared motion estimation models, namely the AuCCA, Random Forest(RF) and the k-means hierarchies (Kmeans). For describing the activities, we used HoG and HoF features for Weizmann and in addition bag-of-words histograms describing the spatio-temporal interest points in a local surrounding of the object for UCF-Sports. To emphasize the efficient representation using AuCCA, we compare training and evaluation time of a cross validation run on UCF-Sports. On average, training CCA and AuCCA takes $32.5 \mathrm{sec}$. and $33.3 \mathrm{sec}$, respectively. In contrast, training a RF with 5 trees of maximum depth 10 takes 1400 sec; training Kmeans with 5 hierarchies with $k=4$ and maximum depth 5 even $4044 \mathrm{sec}$. The evaluation of AuCCA is computed in $0.23 \mathrm{sec}$, where for $\mathrm{RF}$ and Kmeans $9 \mathrm{sec}$ and $20 \mathrm{sec}$ are required, respectively.

\footnotetext{
1 http://www.wisdom.weizmann.ac.il/ vision/SpaceTimeActions.html

2 http://server.cs.ucf.edu/ vision/data.html
} 


\subsection{Unsupervised Adaption via Forward-Backward Regularization}

We tested our forward-backward consistency regularization for the task of unsupervised scene adaption of an activity detector. We trained a walking detector using positive and negative samples from the Weizmann dataset via a linear SVM and tested it on the Weizmann robust dataset 3 This less known dataset contains highly cluttered backgrounds and high irregularities within actions, as can be seen in Figs. 2 and 3, Thus, it is perfectly suited to test our approach for robustness. We compare the performance of the originally trained detector (baseline) with the results of forward-backward consistency checked detections (AuCCA regularized), which shows a clear reduction of false positives. The poor performance of the baseline classifier is induced by the lack of meaningful background samples during training. Consistency checks allow for automatic generation of new positive and especially negative training samples, which are incorporated into the update of the detector via bootstrapping, leading to further enhanced detection results (retrained). The results in Fig. [3] show the capability of AuCCA for mining new training samples by exploiting activity specific motions.
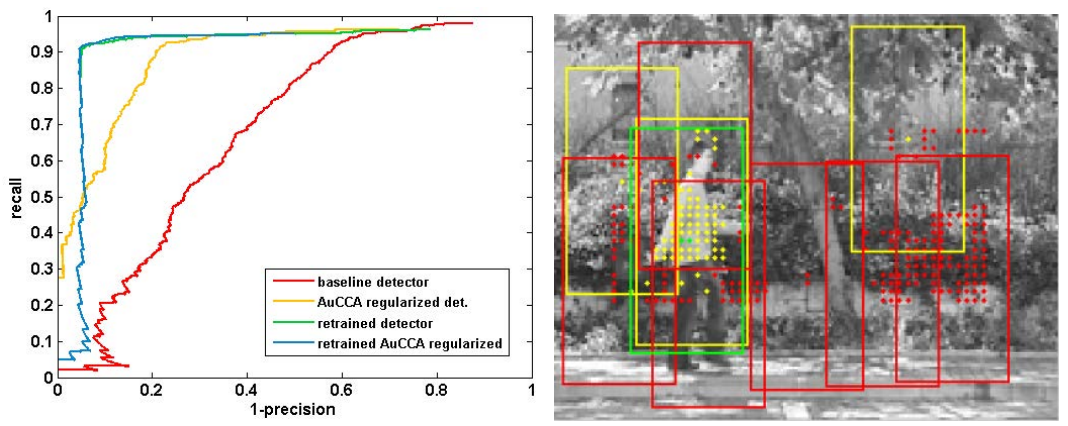

Fig. 3. Results of unsupervised activity detector training using AuCCA forward/backward mapping with $\tau=5$ (left). Examples of positive (green), neutral (yellow), and negative (red) updates for scene adaption from Weizmann to Weizmann robust (right).

\subsection{Comparison of Motion Estimation and Particle Behavior}

The main motivation for this paper was to estimate temporal displacements from high-dimensional training data and to show that this is superior compared to random or optical flow driven motion models. First, we analyze the capabilities of the individual methods proposed in Section 2 compared to optical flow based displacement estimation. Average results for Weizmann and UCF-Sports are shown in Fig. 4. We trained for displacements from $\tau=1, \ldots, 5$ frames, and compare to optical flow with a constant velocity assumption, i.e., the current

\footnotetext{
3 http://www.wisdom.weizmann.ac.il/ vision/VideoAnalysis/Demos/ SpaceTimeActions/DB/robust-deform.zip
} 
flow at time $t$ is propagated linearly by multiplying with $\tau$. We group together results for static activities like wave and bend for Weizmann and golf and lifting for $U C F$-Sports and show individual results for the dynamic activities. One can clearly see that optical flow is not a good choice for linking activity detections over several frames. In contrast, the results of the proposed methods are in the same range with only minor deviations.
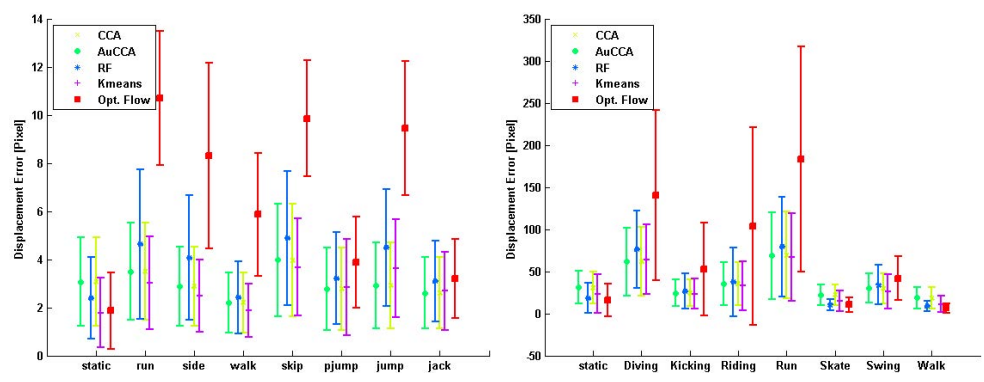

Fig. 4. Average displacement results for Weizmann with $\tau=3$ (left) and for $U C F$ Sports with $\tau=3$ (right)

For evaluating the applicability of the activity linking approach discussed in Section 3 we compared our activity specific displacements learned with AuCCA and a random walk model. We made two tracking runs on UCF-Sports, one with random walk motion model for the particle filter and one with AuCCA motion propositions per particle. To allow for fair comparison, the underlying detector was the same for both runs. This guarantees that particle weights are influenced by the representation. So we only used the displacement values of AuCCA and not the classification results nor the coefficient values. We made two tests with random walk (RW): (a) with set of $N_{P}=100$ particles and $\sigma_{t}=50$ and (b) with $N_{P}=100 \sigma_{t}=200$. The AuCCA particle filter used only $N_{P}=30$ and $\sigma_{d}=25$ to re-sample particles. The average errors to target position for testing on diving, riding, run and swing-side where 15.86pix for the AuCCA, 12.64pix for RW100, and 7.83pix for RW200. This shows that our proposed voting scheme is accurate enough and allows for significant smaller particle sets.

\section{Conclusion}

Most approaches for activity recognition either build on a global image description or assume that the localization of the actor is already given. To overcome these limitations, e.g., if multiple actors are performing multiple actions, combined detection/recognition approaches can be applied. In this work, we addressed this problem by introducing a motion model predicting the spatial displacement over time. In particular, we introduced the idea of augmented Canonical Correlation Analysis (AuCCA), which additionally to image descriptions 
and discriminative class labels incorporates the correlation of the temporal displacement vectors. The proposed AuCCA allows for unsupervised mining new training samples and post-processing of false positive detections by exploiting activity specific motions. Integrated into a particle filter framework the number of required particles can be drastically reduced. We demonstrated our approach for two publicly available standard benchmark datasets, namely Weizmann and UCF-Sports, showing competitive results.

\section{References}

1. Wang, H., Ullah, M.M., Laptev, A.K.I., Schmid, C.: Evaluation of local spatiotemporal features for action recognition. In: BMVC (2009)

2. Kovashka, A., Grauman, K.: Learning a hierarchy of discriminative space-time neighborhood features for human action recognition. In: CVPR (2010)

3. Gorelick, L., Blank, M., Shechtman, E., Irani, M., Basri, R.: Actions as space-time shapes. IEEE Trans. PAMI 29 (2007)

4. Rodriguez, M.D., Ahmed, J., Shah, M.: Action mach - a spatio-temporal maximum average correlation height filter for action recognition. In: CVPR (2008)

5. Lin, Z., Jiang, Z., Davis, L.S.: Recognizing actions by shape-motion prototype trees. In: ICCV (2009)

6. Burgos-Artizzu, X.P., Dollar, P., Lin, D., Anderson, D.J., Perona, P.: Social behavior recognition in continous video. In: CVPR (2012)

7. Yao, A., Gall, J., van Gool, L.: A hough transform-based voting framework for action recognition. In: CVPR (2010)

8. Khamis, S., Morariu, V.I., Davis, L.S.: A flow model for joint action recognition and identity maintenance. In: CVPR (2012)

9. Gall, J., Lempitsky, V.: Class-specific hough forests for object detection. In: CVPR (2009)

10. Hotelling, H.: Relations between two sets of variates. Biometrika 28, 321-377 (1936)

11. Melzer, T., Reiter, M., Bischof, H.: Appearance models based on kernel canonical correlation analysis. Pattern Recognition 36, 1961-1971 (2003)

12. Breiman, L.: Random forests. Machine Learning (2001)

13. Bosch, A., Zisserman, A., Munoz, X.: Image classification using random forests and ferns. In: ICCV (2007)

14. Arulampalam, S., Maskell, S., Gordon, N., Clapp, T.: A tutorial on particle filters for on-line non-linear/non-gaussian bayesian tracking. IEEE Trans. Signal Processing 50, 174-188 (2002) 